\title{
EDITORIAL ANNOUNCEMENT
}

The Cancer Research Campaign has recently announced the affiliation of its scientific journal, The British Journal of Cancer, with two major U.K. scientific societies: the British Association for Cancer Research $(B A C R)$ and the Association of Cancer Physicians (ACP).

The link with the BACR is a natural one, the journal having published the proceedings of its meetings since 1975 with the Campaign additionally supporting many BACR-organized specialist symposia and workshops, etc. A similar link is being established between the journal and the ACP, since its inception in 1985 to provide a forum for the more applied research activities of clinical oncologists.

The British Journal of Cancer has traditionally existed as a medium for the publication of advances in the basic biomedical sciences. As well as promoting laboratory science, it has sought to publicize the achievements of clinical investigators and of epidemiologists in the field. Representation of both fundamental and clinical advances in oncology between the same covers continues to be the primary editorial policy of the journal.

In conjunction with the affiliation with the newly formed ACP, the journal is pleased to announce the appointment, effective from January 1988, of Dr Peter Selby, Senior Lecturer and Consultant in Medicine at the Royal Marsden Hospital and the Institute of Cancer Research, London, in the capacity of Clinical Editor.

Dr Selby joins the Editorial Staff at a time when the number of manuscripts received on all aspects of oncology, but particularly cancer medicine, continue to manifest the upward trend which has been so encouraging and challenging a feature of the last few years.

Contributors to the journal should note that, until further notice, all new manuscripts should continue to be sent to the Editor-in-Chief, Dr Michael Moore, at the Editorial Office in Manchester.

David G. Harnden Chairman, Editorial Board 